\title{
Les coopératives et leur contribution au développement des territoires de l'Outaouais
}

\author{
Guy Chiasson \\ Université du Québec en Outaouais \\ Patrick Duguay et Claudine Lalonde \\ Coopérative de développement régional (CDR) Outaouais-Laurentides
}

\section{INTRODUCTION}

L'objectif de ce texte est justement de montrer la place importante que prend l'entrepreneuriat en économie sociale, incluant les coopératives, dans la trajectoire de développement de l'Outaouais.

La région de l'Outaouais est souvent considérée comme peu dynamique sur le plan de l'entrepreneuriat. Certains commentateurs (Bensouda et Doucet, 2011, p. 88) vont parler d'un « manque de culture entrepreneuriale », un constat partagé par de nombreux acteurs régionaux. Cette faiblesse de l'entrepreneuriat place l'Outaouais parmi les régions québécoises les moins performantes en ce qui a trait à la création de PME. Pour les auteurs, cette faiblesse s'explique par l'omniprésence dans l'économie régionale de grands employeurs - les grandes entreprises de pâtes et papiers jusqu'aux années 1970, et le gouvernement fédéral depuis qui sont en quelque sorte des «rentiers encombrants » (Gaudreault, 2011) et bloquent la création de petites entreprises. Ces constats sont sûrement justifiés lorsqu'on parle de l'entrepreneuriat privé, mais sont peut-être moins pertinents pour ce qui est de l'entrepreneuriat collectif.

L'objectif de ce texte est justement de montrer la place importante que prend l'entrepreneuriat en économie sociale, incluant les coopératives, dans la trajectoire de développement de l'Outaouais. On pourrait déjà citer certaines initiatives innovantes dans leur domaine, et qui ont trouvé leur origine dans la région. En 1974, le premier regroupement de coopératives formé sur la base de l'appartenance régionale allait voir le jour: le Conseil des coopératives de l'Outaouais. Cette première allait paver la voie à un réseau québécois de 11 Coopératives de développement régional
(CDR). Ces CDR regroupent des coopératives issues de secteurs variés, et sont appuyées par le gouvernement québécois pour des interventions de soutien à la création de coopératives. Dans les mêmes années, la Coopérative d'habitation Reboul voit le jour en réaction aux expropriations des résidents de l'Île de Hull, visant à permettre la construction d'un vaste complexe d'édifices fédéraux. Cette initiative citoyenne a créé une des plus grandes coopératives d'habitation du Québec qui, plutôt que de concentrer ses logements en un seul bâtiment, allait essaimer des logements sur l'ensemble du centre-ville en acquérant d'anciennes maisons qu'elle convertit ensuite en duplex ou triplex. La coopérative devint peu à peu un rempart contre la gentrification. C'est aussi à Hull qu'allait naître en 1982 la première entreprise d'insertion du Québec, La Relance. Cette organisation vouée à l'insertion des personnes éloignées du marché du travail constitue encore aujourd'hui un fleuron de l'économie sociale de l'Outaouais avec ses 8 entreprises, ses 70 à 75 personnes en stage d'insertion par année et ses 140 emplois ${ }^{2}$.

Ces quelques exemples illustrent que l'économie sociale est bien présente dans la région depuis plusieurs décennies. D'ailleurs, depuis de nombreuses années, les organisations vouées au développement régional telles que le Conseil régional de développement (CRD) de l'Outaouais et l'organisme qui lui a succédé, la Conférence régionale des élus (CRÉ) de l'Outaouais, ont inscrit l'économie sociale au cœur des priorités du développement régional. L'économie sociale est alors reconnue comme partie intégrante de l'économie de l'Outaouais, et comme outil d'innovation dans la réponse aux besoins et aux aspirations des citoyens et des collectivités. Ces orientations régionales ont contribué à la 
reconnaissance de l'entrepreneuriat collectif par les décideurs et acteurs du développement.

Une première section trace les grandes lignes du portrait global de l'économie sociale en région alors que la section suivante présente deux projets - la Laiterie de l'Outaouais et le marché public de Ripon - qui confirment la capacité des coopératives de l'Outaouais de mobiliser des ressources territoriales pour le développement de la région.

\section{L'ÉCONOMIE SOCIALE EN OUTAOUAIS : NUANCER LE PORTRAIT DE L'ENTREPRENEURIAT}

En 2013, le Pôle d'économie sociale de l'Outaouais commandait un portrait de l'économie sociale de la région (Cholette, 2013). L'étude allait confirmer la présence significative des entreprises d'économie sociale (EÉS) en Outaouais. L'Outaouais comptait alors 327 entreprises collectives, qui se répartissent dans tous les territoires et tous les secteurs d'activité économique. On comptait 119 coopératives et 208 OBNL. On observe une croissance de 23,4 \% entre 2007 (265) et 2012 (327) du nombre d'EÉS. De plus, la part des coopératives s'accroit $5 \%$ plus rapidement que la part des OBNL.

Ces chiffres, qui montrent clairement une croissance des EÉS, contrastent avec l'entrepreneuriat privé où l'Outaouais se positionne plutôt mal face aux autres régions québécoises. En effet, il semble que l'Outaouais comptait, en 2009, 25 PME par 1000 habitants, contre la moyenne québécoise de 37,6 PME par 1000 habitants (Développement économique Canada, 2009). De façon intéressante, la croissance des EÉS place la région de l'Outaouais dans une bien meilleure posture par rapport aux autres régions en ce qui a trait à la création d'entreprises en économie sociale, comme le fait remarquer le portrait sur l'économie sociale en Outaouais : «Ce qui est toutefois occulté, c'est que l'entreprenariat collectif en Outaouais est non seulement performant, mais qu'il surpasse la moyenne québécoise » (Cholette, 2013, p. 45). Ce dynamisme de l'économie sociale en Outaouais est difficile à expliquer précisément, mais il n'est certes pas étranger au rôle actif que jouent les organisations de développement et à des succès de l'entreprenariat collectif, tels que la Laiterie de l'Outaouais, qui ont acquis une grande notoriété.

Une lecture à l'échelle des cinq territoires de MRC de l'Outaouais montre que cette tendance est assez présente un peu partout dans la région. Le Tableau 1 permet de voir que si la majorité des EÉS se trouve sur le territoire urbain de Gatineau, les MRC plus rurales (Vallée-de-la-Gatineau, Papineau et Pontiac) affichent des taux d'EÉS per capita plus élevés que les milieux urbains et périurbains (les Collines-de-l'Outaouais).

Ce dynamisme de l'économie sociale en Outaouais est difficile à expliquer précisément, mais il n'est certes pas étranger au rôle actif que jouent les organisations de développement et à des succès de

l'entreprenariat collectif, tels que la Laiterie de l'Outaouais, qui ont acquis une grande notoriété.

Tableau 1 - Répartition des EÉS dans les différents territoires de la région

\begin{tabular}{|l|l|l|}
\hline & $\begin{array}{l}\text { Nombre d'entreprises d'économie } \\
\text { sociale }\end{array}$ & $\begin{array}{l}\text { Entreprises d'économie sociale } \\
\text { par 10 000 habitants }\end{array}$ \\
\hline Ville de Gatineau & 207 & 8,54 \\
\hline MRC Vallée-de-la-Gatineau & 26 & 12,67 \\
\hline MRC des Collines-de-l'Outaouais & 37 & 14,63 \\
\hline MRC Papineau & 32 & 14,63 \\
\hline MRC Pontiac & 25 & 17,13 \\
\hline Région de l'Outaouais & 327 & 9,57 \\
\hline Ensemble du Québec & 6254 & 8,12 \\
\hline
\end{tabular}


De façon intéressante, nous observons une croissance nettement plus marquée du nombre d'entreprises d'économie sociale dans la MRC Pontiac, qui compte parmi les MRC les plus défavorisées au Québec, ainsi que dans la MRC des Collines-de-l'Outaouais, qui elle trône au sommet des MRC les plus riches. Ce paradoxe nous demanderait des analyses plus pointues pour comprendre le phénomène. Toutefois, la connaissance du terrain nous porte à croire que le recours à l'économie sociale est justifié par la situation socioéconomique de la MRC Pontiac, qui voit exploser les besoins chez une population fragile, alors que le développement de l'économie sociale dans la MRC des Collines-de-l'Outaouais pourrait s'expliquer par son histoire récente et la croissance de population des dernières années. Ces deux cas différents pointent cependant dans une même direction : l'économie sociale a acquis une notoriété importante qui la place au cœur des stratégies de développement des collectivités et des territoires de l'Outaouais.

\section{COOPÉRATIVES ET DÉVELOPPEMENT RÉGIONAL : UNE QUESTION DE RESSOURCES}

Les champions de la croissance en Outaouais :

MRC Pontiac : 108,3 \% (12 EÉS en 2007 à 25 EÉS en 2012)

MRC des Collines-de-l'Outaouais : 76,2 \% (21 EÉS en 2007 à 37 EÉS en 2012)

La première partie de ce texte a permis de faire le portrait de la place que prend l'économie sociale dans l'économie régionale et le développement d'entreprises en Outaouais. Ces constats généraux interpellent des perspectives plus fines permettant d'identifier certains facteurs qui expliquent cette effervescence de l'économie sociale en Outaouais. Plus précisément, nous présentons les résultats de deux études portant sur la contribution des coopératives au développement des territoires. $\mathrm{La}$ première étude portait sur le cas de la Laiterie de l'Outaouais, une entreprise privée, mais qui s'est appuyée dès son démarrage sur deux coopératives (de travailleurs actionnaire et de consommation) (Chiasson et al, à paraître). La seconde étude portait sur le Marché public de Ripon, une petite municipalité rurale située dans la MRC de Papineau.

Ces deux études partageaient une préoccupation commune, celle de mieux comprendre le rapport entre les coopératives et leur territoire. Il s'agissait à travers ces études non seulement de voir dans quelle mesure les coopératives contribuent au développement de leur territoire, mais aussi et surtout de voir les ressources que les coopératives puisent dans le territoire pour assurer leur propre développement.
Cette notion de ressources territoriales (Gumuchian et Pecqueur, 2007) évoquée plus haut nécessite certaines précisions. Jadis, le développement des régions était considéré comme étant principalement l'affaire des grands acteurs que sont les grandes entreprises et l'État, à travers des politiques de redistribution de la croissance (Jean, 1989). Les années 1980 ont montré les limites de ces approches étatiques du développement régional et la recherche de nouvelles façons de faire, s'appuyant sur un rôle plus grand des acteurs du territoire. Certains (Jean, 2006) vont utiliser l'expression développement territorial pour désigner ces nouvelles approches. Dans ce contexte, plusieurs recherches ont fait valoir que les territoires locaux et régionaux recèlent de nombreuses ressources que les anciennes approches avaient négligées. La liste des ressources examinées par les divers courants de recherche inclut des phénomènes comme le capital social (les liens de confiance entre les individus), la culture entrepreneuriale, les réseaux, le capital socioterritorial, etc. Ces phénomènes ne se recoupent pas complètement, mais ils se rejoignent en ce sens qu'ils considèrent tous que la proximité des acteurs sur un même territoire est porteuse d'un lien social fort. Ce lien social entre les acteurs d'un même territoire, ou plutôt les diverses facettes de ce lien social, est 
perçu comme une ressource importante qui assure le dynamisme territorial.

Par notre étude sur deux projets où les coopératives jouent un rôle important, nous espérons apporter des éléments qui permettent de commencer à combler cette lacune.

Cette perspective centrée sur les ressources territoriales a donné lieu à de nombreux travaux sur des régions où l'entreprise privée, et tout particulièrement les petites et moyennes entreprises, occupe une place importante. Au Québec, des régions comme la Beauce et d'autres régions à forte tradition entrepreneuriale privée ont fait l'objet de plusieurs études. Cependant, dans des régions comme celle de l'Outaouais, qui ne sont pas particulièrement reconnues pour leur culture entrepreneuriale, du moins en termes d'entreprises privées, très peu d'études ont permis d'éclairer la question des ressources territoriales et son impact sur le développement de la région. Par notre étude sur deux projets où les coopératives jouent un rôle important, nous espérons apporter des éléments qui permettent de commencer à combler cette lacune. Nous comptons tester l'hypothèse selon laquelle ces coopératives s'appuient particulièrement sur la mobilisation citoyenne comme ressource du territoire (Chiasson et al, à paraître). Nous présentons brièvement chacun de ces projets de développement pour ensuite aborder la question des ressources territoriales dans lesquelles puisent les coopératives.

\subsection{La Laiterie de l'Outaouais ${ }^{3}$}

L'histoire de la Laiterie de l'Outaouais remonte à 2006, au moment où la Laiterie Château de Buckingham, la seule usine de transformation de lait sur le territoire de l'Outaouais, est fermée par son nouveau propriétaire, Agropur. Cette nouvelle a semé la consternation parmi de nombreux acteurs de la région, qui trouvaient inadmissible que le lait produit dans la région de l'Outaouais soit transformé dans les usines d'Agropur à Montréal et que des emplois locaux soient perdus. Un comité de relance piloté par la Coopérative de développement régional (CDR) Outaouais-Laurentides et regrou- pant également la Chambre de commerce de Gatineau et des citoyens est constitué. Un premier scénario de relance était de racheter la vieille usine de Buckingham pour la rénover et la remettre en production. Cette option s'est rapidement avérée impossible puisque la vente de Château à Agropur comportait une entente empêchant que les installations soient utilisées pour produire du lait à nouveau. C'est à partir de ce moment que l'hypothèse de la construction d'une nouvelle laiterie a finalement été retenue par le comité de relance.

Le comité de relance a travaillé conjointement avec deux promoteurs privés prêts à investir dans une nouvelle laiterie en Outaouais. Un de ces promoteurs était propriétaire d'une petite usine de transformation de lait dans la région de MontLaurier; le second détenait une expertise technique importante. Ils pouvaient donc apporter une expertise essentielle au projet. Cependant, le fait de construire une nouvelle laiterie posait un défi financier important. Les promoteurs n'avaient pas les reins assez solides pour réunir les fonds requis pour la construction de cette usine et le projet était jugé trop risqué du point de vue des banques et des organismes gouvernementaux. Le financement du projet est rapidement devenu le problème central pour lequel le comité de relance et particulièrement la CDR ont travaillé d'arrache-pied pendant de nombreux mois. Un répondant confirme le rôle important qu'a joué la CDR dans la relance du projet après un premier échec :

Le partenaire, c'était la CDR, parce que quand personne n'y croyait, il y avait un seul groupe qui travaillait et c'était la CDR [...]. La CDR a mis du personnel, du temps, de l'argent pour faire nos premières publicités. Ils sont allés chercher des petites subventions. Quand le projet aurait dû mourir, il y a eu une période de flottement. Quand on a donné le premier coup et que c'était pour racheter Château, tout le monde était là. Mais quand il a fallu transformer le projet pour la construction d'une usine, donc en ajoutant au moins deux ans à notre scénario, là, c'était la CDR le partenaire. C'était eux qui portaient le projet. (Entrevue L7). 


\section{La présence d'une coopérative de consommateurs permettait déjà de montrer la présence d'un marché pour le lait de l'Outaouais.}

La solution, qui a été construite par le travail patient des acteurs régionaux, est très révélatrice pour nous. Deux coopératives ont été créées : une coopérative de travailleurs actionnaire, regroupant tous les employés qui travailleront éventuellement à l'usine de transformation, et une coopérative de consommateurs. On peut déjà remarquer le caractère innovateur de cet arrangement, où s'unissent une entreprise privée et deux coopératives. La présence d'une coopérative de consommateurs permettait déjà de montrer la présence d'un marché pour le lait de l'Outaouais. Afin de renforcer encore plus l'idée que l'entreprise pouvait être viable, le comité de relance a lancé une campagne, demandant aux citoyens de s'engager en ligne à acheter le lait de l'Outaouais. Cette campagne, appuyée de façon importante par la presse régionale, va donner des résultats qui ont largement dépassé les attentes, avec des engagements de plus de 1,5 million de litres de lait annuellement. Les membres du comité de relance ont fait valoir que cette stratégie a facilité l'obtention des financements requis par les bailleurs de fonds gouvernementaux, notamment Investissement Québec, qui va devenir un des importants partenaires financiers de l'entreprise. La Laiterie de l'Outaouais sera finalement construite et commence à livrer ses premiers litres de lait aux consommateurs de la région en 2010. Un article du journal Le Droit (Bélanger, 2011) estimait que le lait de la Laiterie est bu dans «un foyer sur quatre $\gg$ en Outaouais.

\subsection{Le marché public de Ripon}

Ripon est une municipalité rurale d'un peu plus de 1500 habitants, située dans la MRC de Papineau. Comme bien d'autres municipalités rurales de l'Outaouais, son économie a longtemps été plutôt dépendante de la première transformation des produits forestiers. La fermeture de l'usine de transformation Robert Ribeyron en 2007, le plus gros employeur de Ripon, avait fait craindre pour la vitalité du village. La fermeture $d$ 'autres institutions et commerces locaux comme les bureaux de la Caisse populaire venait renforcer cette crainte, et a amené à la création d'un comité de relance, « un comité de citoyens qui s'est mis sur pied en partenariat avec la municipalité » (entrevue R3). Ce comité, à la suite de deux études, va proposer un plan de revitalisation du cœur villageois dans lequel figure un projet de construire un marché public le long de la Route 317, qui traverse le village. En plus de répondre à un besoin de dynamiser la communauté de Ripon, ce projet aurait aussi un objectif de rapprochement rural-urbain. Comme l'explique une des répondantes :

Dans le milieu rural, on a une grande population à l'été, mais pas le restant de l'année. Tu sais que l'été tu vas avoir ton argent, mais comment tu fais pour arriver le restant de l'année, c'est ça la guerre dans le rural. Dans le marché, c'est la production manuelle, rien d'industrialisé. C'est avec les petits producteurs qu'on va travailler et leur donner une opportunité. Je trouve aussi que c'est une bonne manière de faire connaitre la région, les gens de la ville pourront venir au marché acheter les produits locaux, mais aussi connaitre un peu du rural (Entrevue R1).

Afin d'assurer la construction de l'édifice logeant le marché public et pour l'administrer par la suite, la Coopérative de solidarité Place du Marché a été fondée. La formule de la coopérative de solidarité permettait à de nombreux partenaires impliqués de différentes façons autour du projet de siéger sur le conseil d'administration de la coopérative. C'est le cas de la municipalité de Ripon, des producteurs agroalimentaires ainsi que des citoyens engagés.

\section{La formule de la coopérative de solidarité permettait à de nombreux partenaires impliqués de différentes façons autour du projet de siéger sur le conseil d'administration de la coopérative.}

Comme c'est le cas pour la Laiterie, le financement du projet, en particulier la construction du bâtiment, s'est rapidement avéré un défi important pour les promoteurs du projet. Les répon- 
dants s'entendent pour dire qu'il était important de maintenir les coûts de construction le plus bas possible pour faire en sorte que les coûts de location des espaces du marché soient le plus minimes possible pour les agriculteurs. Un répondant dira à ce sujet :

Si on regarde le coût actuel de location, on devrait le mettre 30 dollars le jour de location par producteur, mais c'était trop cher si on tient compte que la Petite Nation fait partie des gens les plus diminués du Québec et que la plupart des producteurs est arrivé par passion dans la production agricole. Donc, l'objectif d'une production industrielle est écarté, on s'est penché plus sur la production bio et à petite échelle. Donc le schéma qu'on a parti c'était de trouver le maximum de financement sur le bâtiment qui permettrait d'amortir le coût de sortie le maximum possible. (Entrevue R4).

La diminution des coûts de construction du bâtiment aura été rendue possible par la mobilisation de divers partenaires. La municipalité a acheté le terrain et l'a offert en bail emphytéotique à la Coopérative. Des institutions locales comme la MRC, le Centre local de développement et la Caisse populaire ont contribué avec des sommes importantes, alors que le Centre local de développement et la Société d'aide au développement des communautés ont fourni du temps de travail gratuitement pour la préparation du plan de relance ou du plan d'affaires. Finalement, de nombreuses personnes et même des entreprises, notamment de construction, ont fourni du travail bénévole de façon importante. Dans une entrevue réalisée avec le journal Le Droit, Daniel Lavergne, alors président de la coopérative de solidarité, affirme que pour un projet de 700000 \$, seulement 200000 \$ ont été déboursés, le reste a été assuré par des dons et du travail bénévole (Laflamme, 2012). Le marché public a ouvert ses portes au public à l'été 2011.

\subsection{Les ressources territoriales des coopératives}

Les deux cas présentés plus haut prennent place dans des contextes territoriaux en apparence assez différents. Comme notre présentation l'a souligné, la Laiterie de l'Outaouais a élu domicile sur le territoire de la Ville de Gatineau alors que la coopérative Place du Marché a pignon sur rue dans une municipalité rurale périphérique. Ces deux coopératives reflètent assez bien la double réalité territoriale de l'Outaouais que certains (Doucet et al, 2008) ont qualifié de «région qui gagne et qui perd » pour mettre en exergue la cohabitation entre une partie urbaine avec une économie dynamique et tertiarisée et un arrière-pays rural plus fragile. Cependant, au-delà de ces différences, il se dégage tout de même un parcours qui se ressemble. Dans les deux cas, on peut effectivement observer que les promoteurs de projet se sont rapidement rendu compte que les mécanismes habituels de soutien et de développement des projets étaient mal adaptés ou encore ne permettaient pas de répondre aux objectifs visés par les partenaires. Dans le cas de la Laiterie, les bailleurs de fonds habituels (les banques ou encore le gouvernement) se sont montrés réticents à appuyer le projet, le risque étant trop grand. Pour ce qui est de la Coopérative Place du Marché, la construction traditionnelle de l'édifice aurait signifié des coûts de location trop élevés. Les promoteurs ont opté pour d'autres façons de fonctionner qui s'appuient lourdement sur les citoyens comme des partenaires privilégiés dans le développement des projets. Comme nous l'avons expliqué dans le cas de la Laiterie, l'appel aux citoyens consommateurs de lait avait comme objectif de démontrer la viabilité du projet et de défaire les réticences des bailleurs de fonds. À la Coopérative Place du Marché, le travail offert gratuitement par des entreprises et des citoyens emballés par le projet a permis de maintenir les coûts de construction à des niveaux bien en deçà des coûts du marché.

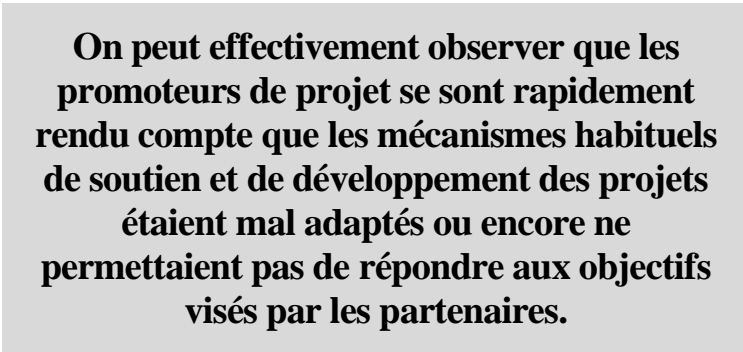

Cette implication des citoyens peut être considérée comme des ressources territoriales des coopératives dans la mesure où elles s'expliquent en bonne partie par une certaine solidarité entre les acteurs d'un même territoire. Le comité de relance de la Laiterie a très explicitement fait référence à l'identité 
régionale dans son effort de mobiliser des consommateurs de lait et d'autres partenaires. Comme en témoignent les propos suivants, l'identité régionale fut également un moteur de l'implication des partenaires :

Personnellement, ce qui m'a poussé [à m'impliquer] c'est la région. Je suis partant du local et de la région. Je suis devenu membre de la coop des consommateurs. Alors au départ, il n'y a pas eu d'hésitation, parce que je crois à la production locale et qu'il faut encourager les gens de chez nous. (Entrevue L1)

L'entreprise continue d'ailleurs de valoriser la production et l'identité régionales à travers son slogan «Ingrédient: lait de chez nous, fierté régionale ». Il semble bien, même si c'est de façon

\section{CONCLUSION}

\section{Nous avons démontré que l'entrepreneuriat en économie sociale de la région est performant, notamment si on le compare avec celui d'autres régions québécoises.}

Ce texte est parti du constat souvent repris, voulant que l'Outaouais figure parmi les régions québécoises où l'entrepreneuriat est très faible. Nous avons voulu montrer que ce constat s'applique moins bien à l'entrepreneuriat collectif, en particulier aux entreprises d'économie sociale. En nous appuyant sur le Portrait des entreprises d'économie sociale en Outaouais, nous avons démontré que l'entrepreneuriat en économie sociale de la région est performant, notamment si on le compare avec celui d'autres régions québécoises. Dans un deuxième temps, en utilisant l'exemple de deux projets de développement impliquant des coopératives, nous avons voulu souligner l'importance d'un facteur qui contribue à expliquer la force de l'entrepreneuriat en économie sociale en Outaouais, soit la capacité d'utiliser la mobilisation citoyenne comme ressource territoriale. L'analyse des cas de la Laiterie et de la Coopérative Place du Marché a effectivement permis de montrer que ces projets ont pu se concrétiser parce que les promoteurs et leur coopérative ont su canaliser et capter le sentiment d'appartenance de la population moins évidente, que la référence à un territoire rural menacé de dévitalisation ait fortement joué dans la mobilisation autour de la Place du Marché4.

De surcroît, il semble que la formule coopérative favorise cette mobilisation citoyenne. Dans les mots d'un des répondants :

Avec ce modèle-là [le modèle coopératif] les gens sont plus portés à s'impliquer parce qu'ils ont la notion que c'est pour tout le monde et ne pas favoriser un seul commerçant sur un autre. Avec les coopératives, on favorise l'ensemble de la municipalité. En parlant des projets, je pense que les coopératives sont la formule gagnante, tu auras plus de gens derrière le projet et tu peux mieux montrer les retombées après. (Entrevue R3)

face au territoire qu'est la région, dans un cas, et la localité, dans l'autre.

\section{BIBLIOGRAPHIE}

Bélanger, Mathieu (2011). «La Laiterie de l'Outaouais a fait sa place », Le Droit, jeudi 16 juin, p. 4.

Bensouda, Reda et Chantal Doucet (2011). Portrait économique de l'Outaouais, Alliance de recherche Université-Communauté Innovation sociale et développement des communautés, en ligne http://uqo.ca/sites/uqo.ca/files/fichiers-uqo/rectorat/cero/ web_-_r44.pdf.

Chiasson, Guy, Sara Léon, Claudine Lalonde et Patrick Duguay (à paraître). "Les territoires de la coopération: le cas de la CDROL », Économie et Solidarités, vol. 42, no 1.

Cholette, Chantal (2013). Portrait des entreprises d'économie sociale de l'Outaouais, Convergence, coopérative d'expertes conseil, 157 pages. http://economiesocialequebec.ca/userImgs/documents /root/documents_gen/OutaouaisPortraitEESmai2013F INAL.pdf.

Développement économique Canada pour les régions du Québec (2015). Outaouais (07) - Profil socioéconomique. En ligne: http://www.decced.gc.ca/fra/publications/economiques/profil/outaou ais/220/index.html.

Doucet, Chantal. Louis Favreau et Martin Robitaille (eds) (2007). L'Outaouais une région qui gagne et qui 
perd, enjeux démographiques et économiques, Gatineau, Chaire de recherche en développement des collectivités.

Gaudreault, Simon (2011). Le syndrome du rentier encombrant : une évaluation de la situation au Canada, mémoire de maitrise, Université du Québec à Montréal, en ligne http://www.irec.net/upload/File/ memoires_et_theses/gaudreaule_rentier20110620_me i_moire_maii_trise_simon_gaudreault_(dei_poi_t_fin al).pdf.

Gumuchian, Hervé et Bernard Pecqueur (eds) (2007). La ressource territoriale. Paris, Économica.

Jean, Bruno (1989). «Le développement régional à l'heure du développement local: le temps des incertitudes", Revue canadienne des sciences régionales, XII, 1, p. 9-24.

Jean, Bruno (2006). «Présentation : le développement territorial: un nouveau regard sur les régions du Québec », Recherches sociographiques, vol. 4, no 3, p. 465-474.

Laflamme, Jessy (2012). « Le couple derrière la place du marché », Le Droit, 22 mai, en ligne http://www.lapresse.ca/le-droit/personnalite/201206/ 12/01-4534149-le-couple-derriere-la-place-dumarché.php.

\section{NOTES}

${ }^{1}$ Nous voulons remercier Sara Léon pour sa contribution à la collecte de données sur laquelle s'appuie ce texte.

${ }^{2} \mathrm{http}: / /$ donnerocharites.ca/fr/LA.RELANCE.OUTAOUAIS INC.._.0_119007011RR0001

${ }^{3}$ Cette section synthétise celle qui est déjà présentée dans Chiasson et al (à paraître).

${ }^{4}$ Voir à ce sujet l'article de Jessy Laflamme (2012) dans Le Droit. 